\title{
Glassy distribution of $\mathrm{Bi}^{3+} / \mathrm{Bi}^{5+}$ in $\mathrm{Bi}_{1-x} \mathrm{~Pb}_{x} \mathrm{NiO}_{3}$ and negative thermal expansion induced by intermetallic charge transfer
}

Kiho Nakano ${ }^{\dagger}$, Kengo Oka\#, $\dagger$, Tetsu Watanuki ${ }^{\S}$, Masaichiro Mizumaki ${ }^{\Delta}$, Akihiko Machida ${ }^{\S}$, Akane Agui $^{\S}$, Hyunjeong Kim ${ }^{\otimes}$, Jun Komiyama ${ }^{\nabla}$, Takashi Mizokawa ${ }^{\perp}$, Takumi Nishikubo ${ }^{\dagger}$, Yuichiro Hattori ${ }^{\dagger}$, Shigenori Ueda ${ }^{\mathbb{I}, \diamond}$, Yuki Sakai ${ }^{*} *$ and Masaki Azuma* ${ }^{*}$

${ }^{\dagger}$ Materials and Structures Laboratory, Tokyo Institute of Technology, 4259 Nagatsuta, Midori-ku, Yokohama, 2268503, Japan

\#Department of Applied Chemistry, Faculty of Science and Engineering, Chuo University, 1-13-27 Kasuga, Bunkyoku, Tokyo 112-8551, Japan

${ }^{\S}$ Quantum Beam Science Center, Japan Atomic Energy Agency, Sayo, Hyogo 679-5148, Japan

${ }^{\triangle}$ Japan Synchrotron Radiation Research Institute, SPring-8, Sayo-gun, Hyogo 679-5198, Japan

${ }^{\otimes}$ National Institute of Advanced Industrial Science and Technology, Central 5, 1-1-1 Higashi, Tsukuba, Ibaraki 3058565, Japan

${ }^{\nabla}$ Department of Complexity Science and Engineering, University of Tokyo, 5-1-5 Kashiwanoha, Kashiwa, Chiba 2778561, Japan

${ }^{\perp}$ Department of Applied Physics, School of Advanced Science and Engineering, Waseda Unversity, 3-4-1 Okubo, Shinjuku-ku, Tokyo 169-8555, Japan

'Quantum Beam Unit, National Institute for Materials Science, Sengen, Tsukuba 305-0047, Japan

${ }^{\bullet}$ Synchrotron X-ray Station at SPring-8, National Institute for Materials Science, Sayo, Hyogo 679-5148, Japan

‡Kanagawa Academy of Science and Technology, KSP, 3-2-1 Sakado, Takatsu-ku, Kawasaki City, Kanagawa, 213-oo12, Japan

\section{Corresponding Authors}

*yukisakai@msl.titech.ac.jp

*mazuma@msl.titech.ac.jp 


\section{Supplementary figures}

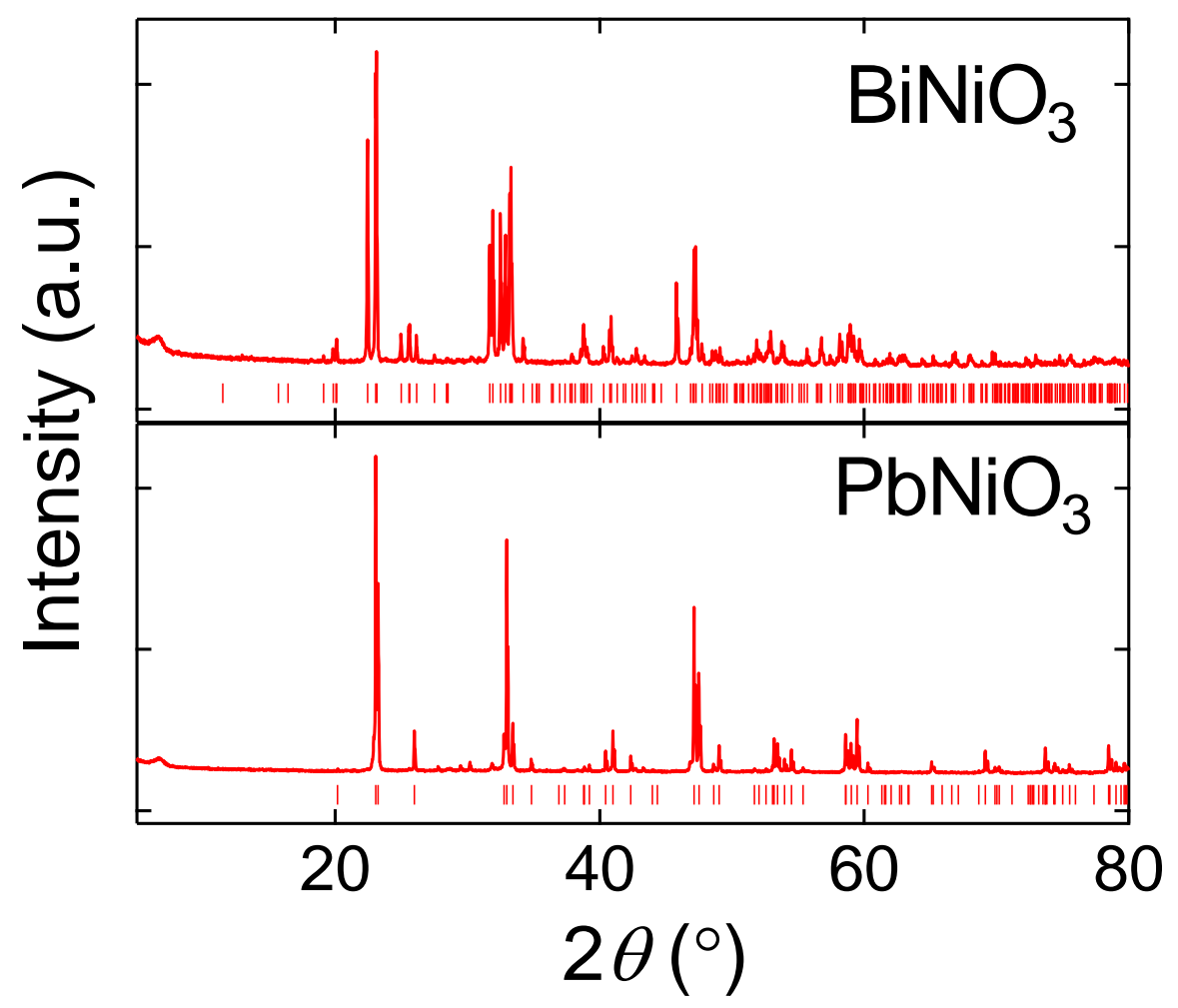

Figure $\mathrm{S}_{1}$ The XRD patterns of $\mathrm{BiNiO}_{3}$ and $\mathrm{PbNiO}_{3}$ samples prepared by the same method described in the text. The tick marks correspond to the positions of Bragg reflections of the $\mathrm{BiNiO}_{3}$ and $\mathrm{PbNiO}_{3}$.

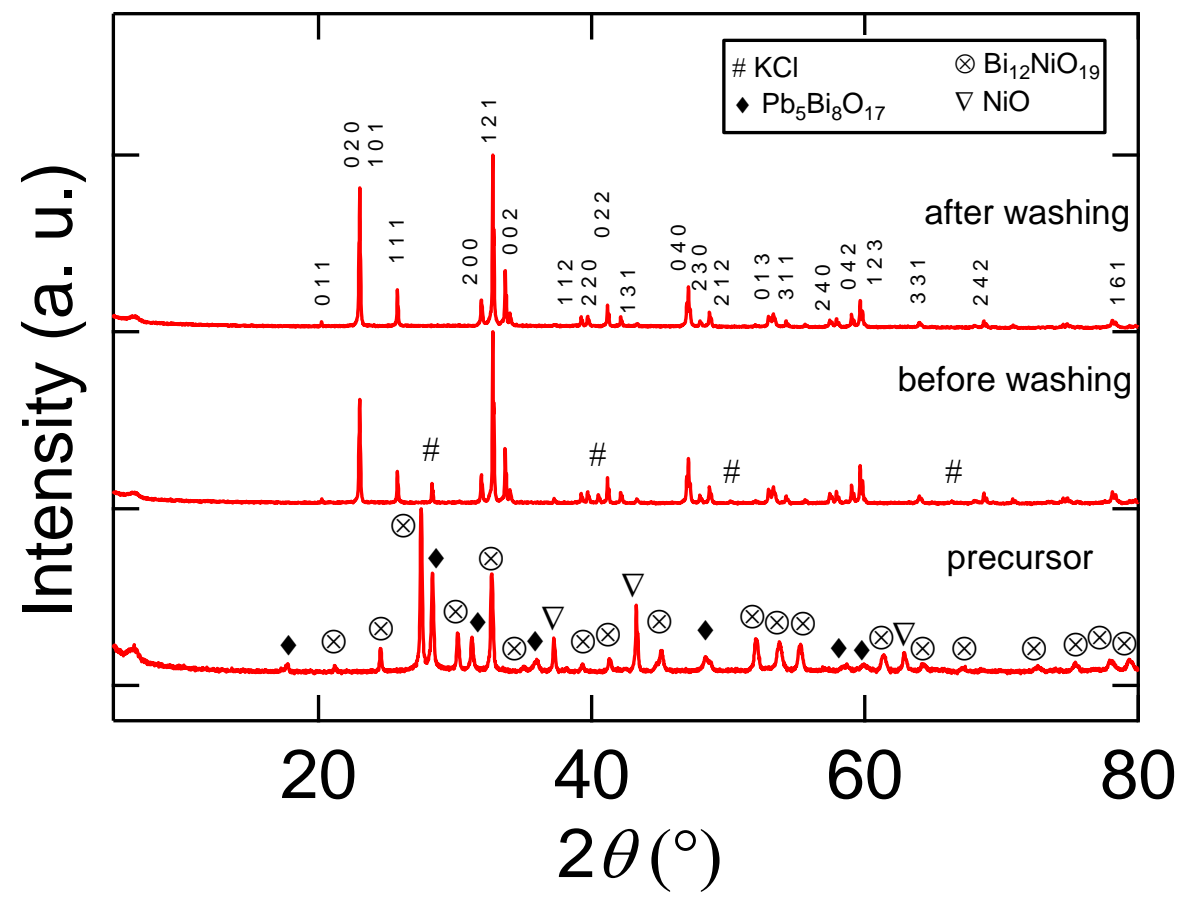

Figure $\mathrm{S}_{2}$ The XRD patterns of the precursor and recovered sample of $\mathrm{Bi}_{0.2} \mathrm{~Pb}_{0.2} \mathrm{NiO}_{3}$ before and after washing. The remaining $\mathrm{KCl}$ was removed by washing with distilled water. 


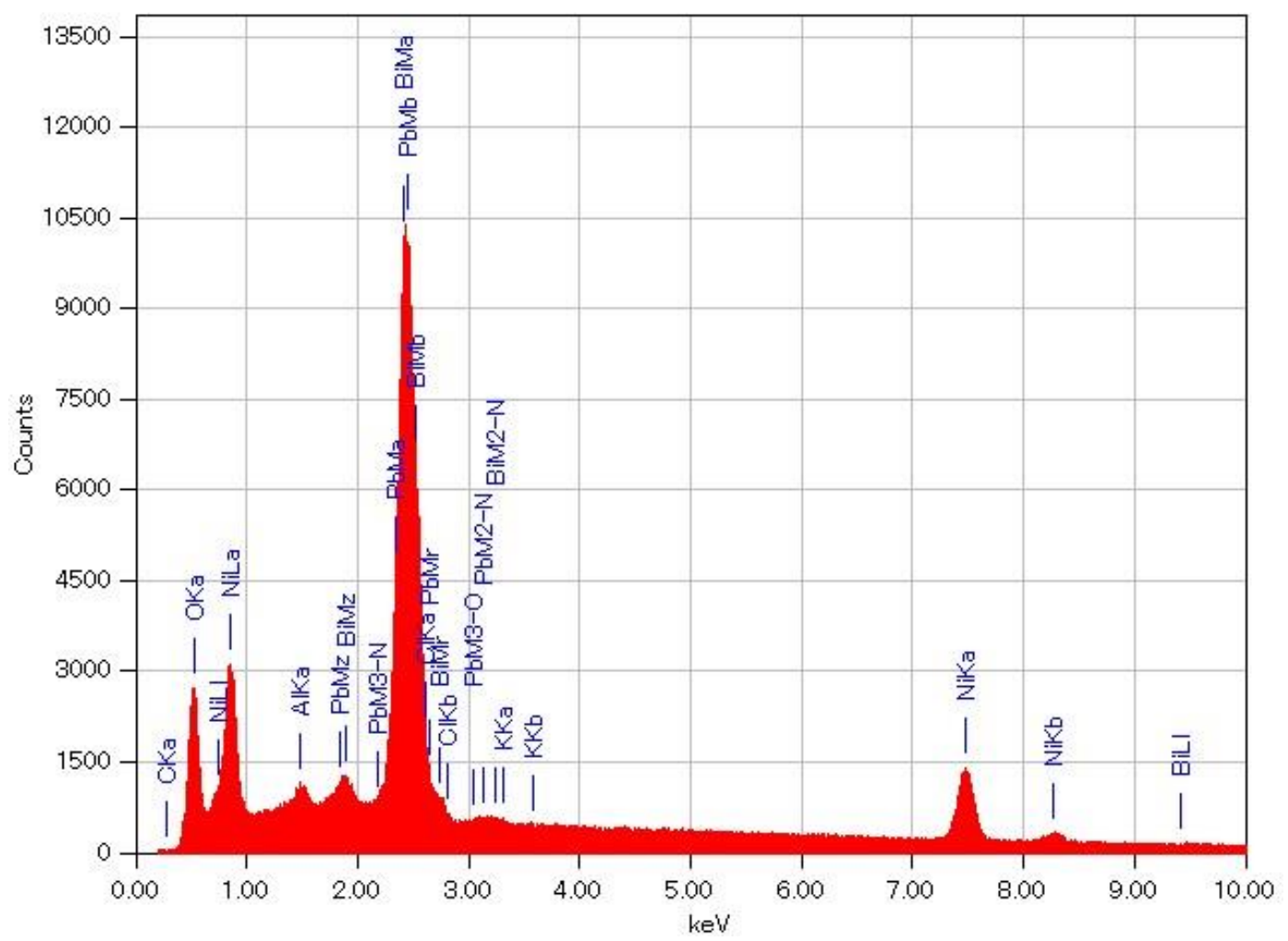

Figure $\mathrm{S}_{3}$ The EDS spectrum of $\mathrm{Bi}_{0.8} \mathrm{~Pb}_{0.2} \mathrm{NiO}_{3}$. The sample was fixed on the holder with a carbon tape containing $\mathrm{Al}$. No trace of residual $\mathrm{C}, \mathrm{N}, \mathrm{Cl}$ and $\mathrm{K}$ was detected. 


\section{Supplementary tables}

Table S1 Fitting results for $\mathrm{Bi} 4 \mathrm{f}$ HAXPES spectra of $\mathrm{Bi}_{0.75} \mathrm{~Pb}_{0.25} \mathrm{NiO}_{3}$.

\begin{tabular}{c|rcc}
\hline Peak No. & Binding energy $(\mathrm{eV})$ & Peak area & FWHM $(\mathrm{eV})$ \\
\hline $\mathrm{Bi}^{5+} 4 \mathrm{f}_{7 / 2}$ & $157.71(0)$ & $0.37(2)$ & $0.68(1)$ \\
$\mathrm{Bi}^{3+} 4 \mathrm{f}_{7 / 2}$ & $158.27(1)$ & $0.96(2)$ & $1.43(1)$ \\
$\mathrm{Bi}^{5+} 4 \mathrm{f}_{5 / 2}$ & $163.02(0)$ & $0.28(1)$ & $0.68(2)$ \\
$\mathrm{Bi}^{3+} 4 \mathrm{f}_{5 / 2}$ & $163.56(1)$ & $0.83(2)$ & $1.50(1)$ \\
\hline
\end{tabular}

Table S2 Fitting results for $\mathrm{Bi}_{4} \mathrm{f}$ HAXPES spectra of $\mathrm{BiNiO}_{3}$.

\begin{tabular}{c|ccc}
\hline Peak No. & Binding energy $(\mathrm{eV})$ & Peak area & FWHM $(\mathrm{eV})$ \\
\hline $\mathrm{Bi}^{5+} 4 \mathrm{f}_{7 / 2}$ & $157.94(0)$ & $0.39(3)$ & $0.69(2)$ \\
$\mathrm{Bi}^{3+} 4 \mathrm{f}_{7 / 2}$ & $158.63(2)$ & $0.85(3)$ & $1.44(3)$ \\
$\mathrm{Bi}^{5+} 4 \mathrm{f}_{5 / 2}$ & $163.25(1)$ & $0.30(2)$ & $0.70(2)$ \\
$\mathrm{Bi}^{3+} 4 \mathrm{f}_{5 / 2}$ & $163.93(2)$ & $0.78(3)$ & $1.54(3)$ \\
\hline
\end{tabular}

Table $\mathrm{S}_{3}$ Fitting results for $\mathrm{Bi} 4 \mathrm{f}$ HAXPES spectra of $\mathrm{BiFeO}_{3}$.

\begin{tabular}{c|ccc}
\hline Peak No. & Binding energy $(\mathrm{eV})$ & Peak area & FWHM $(\mathrm{eV})$ \\
\hline $\mathrm{Bi}^{3+} 4 \mathrm{f}_{7 / 2}$ & $159.08(0)$ & $1.01(1)$ & $1.02(1)$ \\
$\mathrm{Bi}^{3+} 4 \mathrm{f}_{5 / 2}$ & $164.39(0)$ & $0.86(1)$ & $1.09(1)$ \\
\hline
\end{tabular}


Table $\mathrm{S}_{4}$ Fitting results for $\mathrm{Pb} 4 \mathrm{f}$ HAXPES spectra of $\mathrm{Bi}_{\text {o. } 75} \mathrm{~Pb}_{\text {o. } 25} \mathrm{NiO}_{3}$.

\begin{tabular}{c|ccc}
\hline Peak No. & Binding energy $(\mathrm{eV})$ & Peak area & FWHM $(\mathrm{eV})$ \\
\hline $\mathrm{Pb}^{4+} 4 \mathrm{f}_{7 / 2}$ & $137.12(1)$ & $0.85(1)$ & $0.89(1)$ \\
$\mathrm{Pb}^{4+} 4 \mathrm{f}_{5 / 2}$ & $141.98(1)$ & $0.69(1)$ & $0.89(2)$ \\
\hline
\end{tabular}

Table $\mathrm{S}_{5}$ Fitting results for $\mathrm{Pb} 4 \mathrm{f}$ HAXPES spectra of $\mathrm{PbNiO}_{3}$.

\begin{tabular}{c|ccc}
\hline Peak No. & Binding energy $(\mathrm{eV})$ & Peak area & FWHM $(\mathrm{eV})$ \\
\hline $\mathrm{Pb}^{4+} 4 \mathrm{f}_{7 / 2}$ & $137.29(1)$ & $1.13(1)$ & $1.11(1)$ \\
$\mathrm{Pb}^{4+} 4 \mathrm{f}_{5 / 2}$ & $142.15(1)$ & $0.94(1)$ & $1.13(1)$ \\
\hline
\end{tabular}

Table S6 Fitting results for $\mathrm{Pb} 4 \mathrm{f}$ HAXPES spectra of $\mathrm{PbTiO}_{3}$.

\begin{tabular}{c|ccc}
\hline Peak No. & Binding energy $(\mathrm{eV})$ & Peak area & FWHM $(\mathrm{eV})$ \\
\hline $\mathrm{Pb}^{2+} 4 \mathrm{f}_{7 / 2}$ & $138.91(1)$ & $1.22(2)$ & $1.38(2)$ \\
$\mathrm{Pb}^{2+} 4 \mathrm{f}_{5 / 2}$ & $143.76(1)$ & $1.01(2)$ & $1.43(3)$ \\
\hline
\end{tabular}

